Canadian University Music Review

Canadian University Music Review

Revue de musique des universités canadiennes

\title{
Vers une critique musicale socialement fondée
}

\section{John Shepherd}

Volume 10, numéro 2, 1990

Alternative Musicologies

Les Musicologies Alternatives

URI : https://id.erudit.org/iderudit/1014885ar

DOI : https://doi.org/10.7202/1014885ar

Aller au sommaire du numéro

\section{Éditeur(s)}

Canadian University Music Society / Société de musique des universités canadiennes

\section{ISSN}

0710-0353 (imprimé)

2291-2436 (numérique)

Découvrir la revue

\section{Citer cet article}

Shepherd, J. (1990). Vers une critique musicale socialement fondée. Canadian University Music Review / Revue de musique des universités canadiennes, 10(2), 48-67. https://doi.org/10.7202/1014885ar

All Rights Reserved (C Canadian University Music Society / Société de musique des universités canadiennes, 1990
Ce document est protégé par la loi sur le droit d'auteur. L’utilisation des services d'Érudit (y compris la reproduction) est assujettie à sa politique d'utilisation que vous pouvez consulter en ligne.

https://apropos.erudit.org/fr/usagers/politique-dutilisation/ 


\title{
VERS UNE CRITIQUE MUSICALE SOCIALEMENT FONDÉE
}

\author{
John Shepherd
}

... même l'homme blanc qui a plus d'argent et qui est au-dessus de nous tous, chante comme toute autre personne. Quand vous syntonisez la radio, une des premières choses que vous entendez, c'est ses chansons. Il possède tout, toute la connaissance, mais il chante quand même ses chansons. Alors, on devrait se demander ce qui lui est arrivé.

Kuji Iyum musicien Tiv

Ce n'est pas tant à la sociologie, aux rapports sociaux tels qu'on les connaît, de venir expliquer le sens (ou le non-sens) de la musique, c'est plutôt à la musique de nous révéler un social inconnu.

Antoine Hennion

\section{Introduction : trois paradigmes de la musicologie}

Un des débats qui a caractérisé la musicologie historique dans le monde anglo-saxon depuis la deuxième guerre mondiale touche au poids relatif qui devrait être accordé, d'une part, à la détermination, à l'accumulation et à la comparaison des faits, et, d'autre part, à l'entreprise plutôt spéculative d'interprétation et de contextualisation de ces faits en tant que facettes de l'expérience musicale. Sous le climat positiviste de la fin des années cinquante et du début des années soixante, on a mis beaucoup d'accent sur le rôle du musicologue en tant qu'historien établissant des faits sur la base de l'évidence empirique. Ce même historien négligeait ainsi de s'attacher à démontrer ce qui, dans une pièce ou un genre musical donné, provoque un impact à la fois sur les créateurs et sur les auditeurs. Claude V. Palisca, par exemple, a soutenu que:

Si la musicologie doit avoir une importance quelconque parmi les disciplines libérales, il lui faut rencontrer les standards du savoir humaniste. Ces standards sont aussi rigoureux que ceux de la science, mais ils en sont 
distincts. Le document ou l'œuvre authentique est au savoir musical ce que l'expérience contrôlée est à la science. Tout comme la science, qui ne se limite pas à l'évidence acquise par les expériences mais exploite les observations soigneusement mesurées et enregistrées de phénomènes naturels, le musicologue utilise des exemples vivants de la production musicale fidèlement enregistrés et observés (Palisca 1963: 105-106).

En conséquence, le champ de l'esthétique musicale, qui se consacre vraisemblablement à l'investigation et à la compréhension de la logique de l'expérience musicale dans la mesure où cette expérience implique l'esprit et le corps des créateurs et des auditeurs, ne peut accéder au domaine du savoir musical:

On ne peut oublier ... que l'esthétique musicale n'est pas le savoir musical; c'est une expérience musicale et une théorie de la musique qui convergent sur un problème philosophique. L'esthétique ne repose pas sur une évidence documentaire mais sur des principes philosophiques et psychologiques vérifiés par l'expérience (ibidem: 110).

Un raisonnement moins rigoureux a été avancé par Arthur Mendel. Tout en admettant que l'expérience musicale est fondamentale à la motivation de l'historien de la musique, il maintient que plus ce dernier s'éloigne du domaine de l'empirique, plus son entreprise est spéculative et incertaine. La science de la musicologie historique étant débutante, elle devrait, conséquemment, se concentrer sur les faits:

... plus on monte dans l'échelle de la généralité, plus il est difficile de faire ... des tests empiriques ... et plus notre explication assumera le caractère d'une hypothèse; une hypothèse que l'on ne pourrait soumettre aux tests empiriques et qui doit pour toujours rester purement spéculative ... L'histoire de la musique étant une science jeune, elle a encore intérêt, et beaucoup plus que d'autres branches de l'histoire, à établir des faits de base . . . On devrait, pour un certain temps, continuer à s'occuper d'établir ce qu'on appelle des faits individuels ... en travaillant à partir de l'évidence . . . (Mendel 1962: 13-14).

$\mathrm{Au}$ fil des années, pareilles déclarations et d'autres du même genre ont suscité, chez certains musicologues, des répliques dures. Joseph Kerman, par exemple, n'a jamais dévié de son opinion, formulée à l'origine en réponse à Palisca, selon laquelle le but ultime et dominant de la musicologie devrait être la critique:

... la perspicacité critique n'a jamais été facile à définir et a toujours été aussi urgente que problématique. Urgente, parce que la critique est une manière d'interpréter l'art qui essaie de prendre en compte la signification qu'il transmet, le plaisir qu'il procure et la valeur qu'il assume . . . La critique s'occupe des compositions et de l'écoute musicales, des faits et des 
sentiments, de la vie du passé dans le présent, de l'image privée du compositeur dans le miroir public de l'auditoire ... l'étude sérieuse de la critique n'est guère mentionnée par Palisca ... (Kerman 1965: 63).

Il est clair que, jusqu'à maintenant, la critique musicale, celle qui se distingue de l'histoire musicale et de la théorie musicale, a toujours eu à se battre pour se faire reconnaître à l'intérieur de l'entreprise plus large qu'est la musicologie. Au début des années soixante-dix, par exemple, Leonard B. Meyer trouva nécessaire de soutenir ceci:

Découragés et consternés par les incertitudes spéculatives de la théorie, de la critique et, devrait-on ajouter, de l'histoire comme différente de la chronique, trop d'humanistes, particulièrement en musique, ont eu tendance à suivre le sentier battu de l'académisme prudent. Mais choisir la certitude prospective au détriment de la perspicacité présente est à la fois erroné et malencontreux. Cela est erroné parce que la recherche de réponses finales et définitives est un but inatteignable pour les disciplines préoccupées par la compréhension et l'explication. Car, puisque le futur est ouvert et influent, il peut changer notre compréhension et des compositions du passé et des événements historiques du passé. Cela est malencontreux ... parce que les monuments durables du savoir, qui ont formé les idées et les croyances des humains, loin d'être circonspects, ont été ceux qui ont éclairé une relation, une œuvre d'art ou une époque par le biais d'une hypothèse audacieuse et étoffée (Meyer 1973: 25).

$\mathrm{Si}$, en musicologie, la démarche interprétative et critique a dû résister fermement au poids de l'empirisme et du positivisme, alors les approches musicologiques qui ont tenté d'englober les leçons des sciences sociales ont été forcé d'adopter une position encore plus périphérique. Il y a plusieurs années, la discipline de l'ethnomusicologie reconnaissait, à travers différentes formulations, les liens étroits unissant «musique » et «culture » ou «musique » et «société ». Mais, comme Richard Leppert et Susan McClary l'ont récemment observé:

Dans la plupart des cas ... les découvertes de l'ethnomusicologie n'ont été acceptables pour la musicologie historique que dans la mesure où elles concernent d'autres cultures. Autrement dit, reconnaitre que d'autres musiques sont liées aux valeurs sociales ne mène pas nécessairement à la conclusion qu'il doit également en être ainsi de notre musique; le plus souvent, cela conduit simplement à la réaffirmation chauviniste et idéologique de la supériorité de l'art occidental, tenu pour autonome (Leppert et McClary 1987b: xviii).

Toutefois, le reniement des leçons des sciences sociales en général, et de quelques formes de l'ethnomusicologie en particulier, prend parfois une route plus subtile que celle sous-entendue par Leppert et McClary. Réagissant à l'empirisme positiviste et socialement décontextualisé qui, à la fin des années 
cinquante et au début des années soixante, dominait la musicologie, Frank Ll. Harrison plaida pour une démarche sociologiquement fondée de la musicologie. Pour Harrison, le but ultime de la musicologie devint:

l'étude des hommes en société en autant qu'ils s'expriment par l'intermédiaire de la musique ... De ce point de vue, c'est la fonction de toute musicologie d'être ethnomusicologie, c'est-à-dire d'inclure dans son champ de recherche du matériel dit «sociologique» (Harrison 1963: 79-80).

Si Kerman fut capable de «consentir à cette formulation générale » (1965: 62), il était néanmoins en faveur d'un changement d'intérêt. Ce changement:

... traite le même matériel et utilise les mêmes mots. Cependant, les œuvres d'art ne sont pas étudiées comme des moyens de faire avancer «l'étude des hommes en société ». Il faut juste inverser les termes: les hommes en société sont étudiés pour faire avancer la compréhension des œuvres d'art. On peut dire qu'il s'agit ici d'une orientation critique, pour la différencier de l'orientation sociologique (ibidem).

La distinction faite par Kerman est cruciale pour la musicologie. En même temps, c'est une distinction qui permet fréquemment de considérer comme un peu plus que des symboles les références à la pensée socio-scientifique. Ainsi Kerman peut-il affirmer que, dans sa conception de la musicologie, «l'histoire et la sociologie ne sont pas évaluées comme des fins, mais comme des moyens» (ibidem), et que:

chacune des choses que nous faisons - paléographie, transcription, études de répertoire, travail archivistique, biographie, sociologie, Auffuhrungspraxis, écoles et influences, théorie, analyse stylistique, analyse individuelle chacune de ces choses, que certains traitent comme une fin en soi, est traitée comme un échelon ... le haut de l'échelle donnant accès à une plate-forme de perspicacité envers les œuvres d'art individuelles ... (ibidem: 62-63).

Il arrive souvent que toutes ces choses fassent partie de l'entreprise musicologique, excepté la pensée sociologique et socio-scientifique. Pareille omission, dans les fondations mêmes de la musicologie critique, a incité une chercheure comme Rose Rosengard Subotnik, dont le travail a constamment été façonné par la théorie critique, à blâmer vertement les accomplissements de la critique musicale américaine:

... la critique musicale américaine est un phénomène évasif et fragmentaire. En majeure partie, elle consiste en essais éclectiques et très contradictoires, écrits par des universitaires qui, bien rarement, identifient leur entreprise de «critique» et qui, en outre, travaillent de manière relativement isolée étant donné que, dans les départements de musique américains, on ne trouve presque jamais plus d'un seul de ces essayistes, qu'une minorité de leurs 
étudiants seulement peut se permettre de rester dans le domaine de la critique et que très peu d'entre eux ont engendré une école de méthodes ou de pensée critique qui soit le moindrement identifiable . . . collectivement, ils ont eu un impact relativement faible sur le caractère et la direction de la musicologie américaine dans son ensemble institutionnel (Subotnik 1982: 146).

Kerman a le sentiment que «. . . collectivement, nous avons fait un peu mieux que ça » (1985: 148). Mais, symptomatiquement, Kerman observe que l'ethnomusicologie et les sciences sociales ont un rôle limité dans la pratique de la musicologie critique:

Si les méthodes de l'ethnomusicologie n'ont pas pris ... ce n'est pas par manque d'efforts. La raison de cet échec relatif me semble claire. Il n'y a qu'un nombre limité de domaines ... où la recherche ethnomusicologique puisse empiéter directement sur l'étude de la musique occidentale. La musique occidentale est tout simplement trop différente des autres musiques, et ses contextes culturels sont aussi trop différents des autres contextes culturels. La musicologie s'est traditionnellement allié les disciplines humanistes, et non les sciences sociales . . . Cette alliance est encore la meilleure base pour développer une structure contextuelle de la musique occidentale . . . C'est une chose . . . que de se baser sur l'historiographie moderne, grandement influencée par l'anthropologie et la sociologie, et c'en est une autre que de se baser directement sur ces disciplines dans la compréhension de la musique occidentale (ibidem: 174-175).

L'on pourrait rétorquer que la question centrale de la musicologie occidentale à l'heure actuelle n'est pas celle de l'importance relative qui devrait être attribuée, d'un côté, au travail empirique et, de l'autre, à l'entreprise critique, mais celle de la nature même de l'entreprise critique. Il ne s'agit pas ici de dévaloriser le travail empirique ou de suggérer qu'il est intellectuellement moins valable et moins légitime. La musicologie en tant que critique, peu importe l'orientation de cette critique, ne peut tout simplement pas procéder sans l'évidence provenant de sources premières. Les critiques de Subotnik envers plusieurs de ceux qui font un travail empirique aux États-Unis sont politiquement acceptables, et il en va de même de ses jugements sur le positivisme en tant que base intellectuelle d'un travail empirique. Mais accorder trop d'attention à l'empirisme positiviste, "voir une impasse», comme l'a exprimé Kerman, "entre la critique et son antithèse, la musicologie positiviste» (ibidem: 148) nous distrairait de discussions plus importantes. S'il est possible de n'être pas d'accord avec Kerman lorsqu'il écarte, bien légèrement, les contributions que pourraient apporter à la musicologie critique l'ethnomusicologie et les sciences sociales, alors il est également possible de n'être pas d'accord avec Subotnik à deux égards. Premièrement, elle ne semble pas explorer la possibilité de recontextualiser les résultats empiriques du positivisme à l'intérieur de perspectives plus critiques, non plus, bien sûr, que d'établir des «agendas » empiriques à la lumière de telles 
perspectives. Ceci explique peut-être pourquoi Subotnik considère comme tellement limités les accomplissements de la musicologie critique aux États-Unis. Deuxièmement, on peut supposer que Subotnik ne distingue pas très clairement le type de critique effectué par des personnalités telles que Charles Rosen, Joseph Kerman, Leonard B. Meyer, Edward T. Cone, Leo Treitler et Edward Lippman, critique façonnée par une conception humaniste, de celui pratiqué par Theodor W. Adorno, Carl Dahlhaus, Jean-Jacques Nattiez et Roland Barthes, qui s'avère habité de notions sociologiques et sémiologiques. Tout dépend du sens que l'on donne au mot «critique ". Tout dépend également de la légitimité que l'on accorde à la distinction qualitative, sous-entendue par Kerman entre la musique occidentale (dite « sérieuse») et les autres musiques du monde.

\section{Les études sur la musique populaire: progrès et limitations}

C'est dans le cadre des études sur la musique populaire qu'ont été traités avec le maximum d'acuité les problèmes liés au développement d'une critique musicale socialement fondée. Le plus souvent, les esthéticiens et les musicologues ont approché les questions d'esthétique et de valeur d'une manière qui dégage la musique des circonstances sociales et historiques de sa création et de son appréciation. En effet, dans l'évaluation de la musique «sérieuse», on a pris pour une marque de qualité le fait qu'elle soit perçue comme a-sociale, sans taches et non affectée par des forces sociales. D'autre part, les esthéticiens et les musicologues ont traditionnellement considéré la musique «populaire» comme moins valable, précisément parce qu'il n'est pas possible de nier aisément les significations sociales inhérentes à plusieurs de ses genres. Jusqu'à maintenant, les chercheurs en musique populaire ont dû envisager deux questions: comment développer une compréhension des significations sociales, culturelles et personnelles intrinsèques à la musique populaire, et comment développer une structure de critique et d'évaluation de la musique populaire qui tienne compte des spécificités de cette même musique tout en lui conférant un statut d'objet aussi digne d'étude que d'autres sortes de musique.

Jusqu'à très récemment, les études en musique populaire étaient surtout axées sur la première de ces deux questions. Il est clair que le développement d'une structure de critique et d'évaluation ne peut avancer en dehors d'une compréhension des significations impliquées par la musique que l'on veut critiquer et évaluer. Outre cela, toutefois, des problèmes importants sont apparus lorsqu'on a essayé de comprendre la signification sociale, culturelle et personnelle de la musique populaire, parce qu'aucune des deux disciplines principales qui ont contribué à l'étude de cette musique, la sociologie et la musicologie, ne s'appuyait sur des protocoles théoriques permettant de déterminer les significations de la musique populaire (Shepherd 1982: 
145-149; Shepherd 1985). De plus, la tentative d'élaboration de ces protocoles a, plus souvent qu'autrement, dévoilé les préjugés disciplinaires des individus chercheurs. Alors que les sociologues ont eu tendance à croire qu'il était possible de trouver des réponses à la compréhension des significations de la musique populaire à l'intérieur de processus contextuels (processus sociaux, historiques, culturels, économiques, politiques, psychologiques et biographiques qui, pour être extrinsèques à l'événement musical, ne l'imprègnent pas moins de signification pour les gens), les musicologues ont plutôt été portés à penser que de telles réponses devaient provenir de l'examen de processus textuels (processus sonores, de mouvements, verbaux et visuels, intrinsèques à l'événement musical mais néanmoins imbriqués aux processus contextuels).

Discutant des tâches respectives de la musicologie historique et de l'analyse musicale, Kerman a bien reconnu l'importance de ces deux dimensions que sont le processus musical et l'expérience musicale: «là où l'attention de l'analyste se concentre sur le fonctionnement interne d'un chef-d'œuvre,» dit-il, «celle du musicologue se porte sur un réseau de faits et de conditions qui s'y entrechoquent» (Kerman 1985: 72). Par ailleurs, la contribution qu'une synthèse de la musicologie historique et de l'analyse musicale aurait pu apporter à la compréhension de la musique a été compromise par l'importance qu'on a traditionnellement accordée au néopositivisme. «Il est certain », observe Kerman:

... qu'on a déjà eu l'occasion de voir jusqu'à quel point les musicologues, en général, ont échoué ... Pendant la période de l'après-guerre, surtout, leur concentration sur des tâches positivistes limitées a eu l'effet décisif de mettre à l'écart «la musique elle-même»; trop souvent leur approche des æuvres musicales a paru irréfléchie, décevante et superficielle (ibidem: 72).

Cependant, continue-t-il, «... si la faille caractéristique des musicologues est la superficialité, celles des analystes est la myopie»:

En isolant la partition de son contexte, de façon à l'examiner comme un organisme autonome, l'analyste soustrait cet organisme de l'écologie qui le soutient. Il semble à peine possible, aujourd'hui, d'ignorer ce fait (ibidem: 73).

Puisque le néopositivisme a maintenu une séparation entre les intérêts traditionnels de la musicologie historique et ceux de l'analyse musicale, ce sont les études en musique populaire qui ont pris en charge les rôles respectives du texte et du contexte dans la production de la signification musicale. Toutefois, si des mouvements de réconciliation et d'intégration d'intérêts textuels et contextuels ont été rendu possibles dans les études en musique populaire par le biais d'un fondement social, la façon dont ce fondement social doit être conceptualisé reste problématique. Car, à 
l'intérieur des études en musique populaire subsistent des tensions, semblables à celles rencontrées non seulement entre la musicologie historique et la théorie musicale, mais aussi entre les ethnomusicologues aux tendances anthropologiques et ceux aux tendances musicologiques.

Un récent article de Simon Frith (1987) illustre bien la nature de ces tensions. L'article est capital en ce que les questions touchant l'esthétique et la valeur de la musique populaire sont clairement considérées comme possédant une importance primordiale, et cela, pour la première fois dans le développement de cette sous-discipline. Et ces questions sont traitées en accord avec la volonté des études en musique populaire d'ancrer les analyses dans l'univers social. C'est dire que Frith n'envisage pas les problèmes d'esthétique et de valeur de façon isolée. Il se soucie plutôt de fonder ces questions sur la perception des consommateurs. Il cherche à savoir pourquoi les "gens ordinaires» aiment et valorisent certaines musiques plus que d'autres, et à découvrir ce qui fait que certaines musiques «communiquent » alors que d'autres n'y parviennent pas.

Selon Frith, les personnes aiment la musique à la condition qu'elle soit perçue comme transcendant les forces sociales de sa propre création:

... les gens entendent la musique qu'ils aiment comme quelque chose de spécial ... elle semble donner une expérience qui transcende le monde, qui nous transporte "hors de nous-même» . . . cette chose unique . . . nous libère des routines et attentes quotidiennes qui emcombrent nos identités sociales ... si nous croyons posséder notre musique, nous nous sentons souvent possédés par elle. La transcendance est ... tout autant une partie de l'esthétique de la musique populaire qu'elle l'est de l'esthétique de la musique sérieuse (1987: 144).

D'après Frith, cependant, «la transcendance ne signale pas la libération de la musique des forces sociales, mais, au contraire, le façonnement de la musique par ces forces». Ce qu'on devrait observer, selon lui, «c'est . . . comment . . . une pièce musicale établit, en tout premier lieu, l'idée de la vérité - la musique populaire définissant elle-même ses propres standards esthétiques » (ibidem: 137). La musique populaire construit donc l'univers des personnes plutôt que de le refléter. La conscience et la subjectivité se créent à travers des pratiques signifiantes et «. . . les besoins et attentes culturels sont . . . matériellement fondés » (ibidem: 144). Ainsi, en pénétrant dans le domaine de l'esthétique et de la valeur en musique, Frith reconnait-il l'effet individualisant de la musique, sa capacité de former, chez un individu, la base de sa négociation d'une situation sociale et d'une réalité sociale prise pour «donnée». «La musique nous met certainement à notre place,» dit Frith, «mais elle peut aussi suggérer que nos circonstances sociales ne sont pas immuables ... La musique populaire n'est en elle-même ni révolutionnaire ni réactionnaire. Elle est une source de sentiments profonds qui, parce 
qu'ils sont socialement codés, peuvent se retourner contre le 'sens commun' » (ibidem: 149).

Frith ne nie donc pas, en fin de compte, la portée de l'autonomie individuelle, si chère aux discussions traditionnelles sur l'esthétique et la valeur dans le domaine de la critique de la musique "sérieuse». Chaque ouvre et chaque genre de musique, ainsi que les subjectivités de l'origine de leur création et de leur appréciation, ne sont pas vus comme des «calques» ni ne se réduisent à un commentaire idéologique passif sur la surface de la vie sociale. Ce que Frith a réussi à faire, c'est de recontextualiser les notions et les intérêts traditionnels de la critique de la «haute culture » dans le sens de la médiation sociale et historique de toute vie et de toute réalité. Cet accomplissement rehaussera sûrement la démarche de la critique musicale en permettant aux musiques «sérieuse » et "populaire» d'être traitées de façon similaire, ceci facilitant à son tour l'effondrement partiel de catégories qui, à leur meilleur, ne sont que des étiquettes confortables. C'est à la nature de cet accomplissement que Leppert et McClary font référence lorsqu'ils disent:

Traditionnellement, on a considéré la musique populaire comme utilitaire, dépourvue de valeur esthétique, tandis que la musique «sérieuse» était perçue comme totalement esthétique, exempte d'utilité, de but, de propos. L'effondrement de ces catégories conventionnelles a plusieurs implications méthodologiques. Leur transmission requiert une justification historique de la fonction idéologique qu'elles possèdent et elle suggère aussi - ou même exige - un croisement de méthodes: c'est dire que la dimension esthétique de la musique populaire devient visible en même temps que les fonctions sociales et politiques de la musique «sérieuse » deviennent inévitables (Leppert et McClary 1987b: xv-xvi).

En dépit de ces propositions toutefois, il semble subsister dans la ligne de pensée de Frith une tension entre les «besoins et attentes culturels " comme étant «matériellement fondés» et l'effet individualisant de la musique populaire.

Ainsi, il est intéressant de constater à quel point l'insistance de Frith sur le rapport entre les questions d'esthétique et de valeur et celles de l'individualisation des gens à travers la musique se rapproche de la théorie esquissée, il y a quelque trente ans, par Leonard B. Meyer (1959). Cependant, la différence cruciale entre Frith et Meyer réside dans le fait que là où Meyer s'est servi d'arguments concernant l'individualisation des gens par la musique dans le but de rejeter certaines musiques populaires, Frith s'en sert maintenant pour démontrer la légitimité esthétique de ces musiques. Néanmoins, il y a une ambivalence ici, et cette ambivalence a trait à la nature précise de la dialectique entre les processus par lesquels la musique populaire construit les identités et les processus par lesquels les personnes s'individualisent à travers la musique populaire. Il devient alors difficile de 
voir exactement comment « la transcendance [à travers la musique populaire] ne signale pas la libération de la musique des forces sociales, mais, au contraire, le façonnement de la musique par ces forces " (Frith 1987: 144).

Il est clair que les réponses à de telles questions ne découleront pas uniquement de la spéculation théorique. Une expérience de terrain, menée dans la ville de Montréal par Jennifer Giles, par exemple, a démontré que les relations passives et actives à la musique populaire, ainsi que la perception consciente et «inconsciente» des messages sociaux et culturels qui y sont contenus, varient de manière significative entre des individus occupant la même position dans la structure sociale, en rapport avec les différents paramètres contextuels et textuels de la musique et en rapport, également, avec la façon dont ces paramètres sont interprétés (Giles 1987). Les processus de reproduction et de résistance à travers la musique sont très complexes et s'appuient, en dernière instance, sur des considérations biographiques individuelles. Le rapport complexe des individus à l'usage et à la consommation qu'ils font de la musique constitue et reflète une logique cohérente, une logique de la pratique enracinée dans les processus biographiques ainsi que dans les processus d'internalisation variable de conditions sociales objectives et d'identités culturelles associées à ces dernières.

Ce qu'il reste à théoriser, ce sont les processus par lesquels les gens en viennent à s'approprier la musique, par des moyens qui sont socialement reproductibles, socialement résistants, socialement créatifs et socialement individuels, mais qui, en même temps, se conforment à la logique des processus biographiques par lesquels les individus ont été socialement constitués et négocient cette logique (Giles et Shepherd 1989). En d'autres mots, il faut développer un cadre théorique qui tienne compte de la position de Frith, selon laquelle les «questions esthétiques » doivent être traitées à partir d'une «base sociologique» (1987: 144), en même temps que de son affirmation selon laquelle «la musique nous met certainement à notre place ... Mais elle peut aussi suggérer que nos circonstances sociales ne sont pas immuables » (ibidem: 149). Une telle théorisation requiert à son tour une conceptualisation particulière de l'articulation musical/social. Ce qui importe alors, dans le domaine des études sur la musique populaire, c'est le rétablissement de la musique comme lieu d'expression individuelle et collective, rétablissement qui reconnaisse et le fondement social de toute expérience musicale et les spécificités de la musique en tant que texte dans leur capacité de faciliter et de refléter les processus, socialement médiatisés, d'individualisation, d'identification et de créativité.

\section{La spécificité du texte musical}

Dans une critique bien fondée d'un de mes articles, Keith Swanwick affirmait que: 
Même si la "signification» de la musique résulte d'un contexte social, on ne peut la relier, en dernière instance, à la signification sociale. Car si tel était le cas, il deviendrait impossible de voir comment quiconque peut réagir à la musique d'autres cultures ou d'autres époques et trouver cette musique significative, efficace, troublante, émouvante . . . saisir les déviations de la normalité, les caractéristiques particulières et personnelles d'un compositeur ou d'un exécutant. C'est à ces choses que l'on est capable de se rattacher, à travers le temps historique et la différence culturelle, et c'est tout cela qui rend possible notre réaction à une musique provenant d'une structure sociale étrangère, malgré notre ignorance presque totale de cette structure (Swanwick 1984: 53).

Graham Vulliamy et moi répliquâmes à cette critique de la façon suivante:

On ne peut nier le fait que nous réagissions à la musique d'autres cultures. Cependant, la question cruciale est de savoir si la réaction est fidèle à la musique et à la culture. Même à l'intérieur de notre propre société, il y a plusieurs évidences démontrant que les membres d'une culture peuvent mal interpréter la musique d'une autre culture, même quand l'interprétation est sensible et bien intentionnée ... Pareilles erreurs d'interprétation ne peuvent être évitées que si l'on examine les musiques d'autres cultures comme des aspects constitutifs des sociétés dans lesquelles elles sont créées et interprétées. Séparer la créativité individuelle du processus social pour approcher, d'une façon trans-culturelle, la signification musicale de cette créativité, c'est permettre à l'idéalisme et à l'ethnocentrisme de s'introduire par la porte arrière (Vulliamy et Shepherd 1984: 63).

Même si, aujourd'hui, j'endosserais encore cette réponse, je crois néanmoins que Swanwick a mis en relief, dans sa critique, quelque chose d'important que ni Vulliamy ni moi n'avons reconnu de façon explicite. Bien que ni Vulliamy ni moi n'ayons jamais souscrit à une vision de la musique qui soit déterminée par le social, je pense qu'il serait juste de dire que nous avons eu tendance à reproduire la présomption, souvent ignorée, des analyses britanniques des sous-cultures, présomption selon laquelle, en dernière instance, il faut comprendre la signification de processus musicaux spécifiques comme ancrée dans la réalité sociale, qui est à la fois extrinsèque et intrinsèque aux dits processus. Ce que cette prise de position met de côté, c'est, du fait de la relative autonomie (du moins, en théorie) de tous les domaines du processus social, la possibilité d'envisager l'existence d'articulations musicales/sociales qui soient purement intrinsèques au processus musical, de sorte que toute signification extrinsèque ne puisse résulter que de telles articulations, lesquelles seraient subséquemment reconnues comme pertinentes à d'autres domaines du processus social. Autrement dit, la signification en musique ne dépend pas, en fin de compte, de l'existence d'échos venant d'autres domaines du processus social, échos d'articulations qu'il s'agirait ensuite d'exprimer musicalement. En ce sens, il demeure 
possible pour les individus de créer et de connaître des aspects de leur univers auxquels ils ne sauraient accéder autrement que par l'intermédiaire de la musique.

L'importance de ce point s'amplifie lorsqu'on réalise qu'il n'existe aucune réalité sociale à laquelle la musique contribue, qui n'ait de limites fixes. Dans la mesure où la musique d'une culture peut transmettre à cette culture quelque chose qu'elle ne désire pas entendre ou reconnaitre, on peut penser que la musique n'existe que dans les fentes et les marges ou au-delà des limites de ce qui passe pour la "réalité » de cette culture. Dans plusieurs cultures, la musique semble agir comme une forme de médiation entre le connu et l'inconnu, l'acceptable et l'inacceptable, ce qui est puissant et ce qui est dangereux. On peut alors se demander quelle est la différence de statut, pour ce qui concerne les possibilités de connaissance et d'apprentissage, entre une musique qui est «intrinsèque» à une culture et une musique qui lui est «extrinsèque ». S'il y a une différence, c'en est peut-être une de degré plutôt que de nature, dans le sens où certaines musiques sont plus proches que d'autres de la «réalité sociale» spécifique à laquelle elles s'adressent.

Il est donc possible d'être d'accord avec Swanwick lorsqu'il dit que «les musiques d'autres cultures ou d'autres époques [peuvent être] significatives, efficaces, troublantes, émouvantes . . .» Non pas parce qu'il y a un élément de créativité individuelle qui existe sur une base trans-culturelle, indépendamment de contextes sociaux spécifiques, et non plus parce que la communication trans-culturelle de la musique donne lieu à des interprétations déformées (quoique l'interprétation de la musique d'une culture par une autre entraîne certes des questions complexes et difficiles). Les musiques d'autres cultures et d'autres époques peuvent être significatives, efficaces, troublantes et émouvantes parce qu'elles ont quelque chose de pertinent à dire. Ceci est encore plus vrai, peut-être, dans les cultures du capitalisme industriel, où les réalités sociales ont, d'une façon ou d'une autre, relégué la musique dans les fentes et les marges en tant que «capital culturel», «loisir» ou «divertissement ».

En effet, il est possible de dire qu'une certaine surdité a surgi dans les cultures du capitalisme industriel par rapport à ce que notre musique peut nous révéler de nous-mêmes, et cela, parce qu'on utilise généralement la musique pour échapper aux conséquences quotidiennes de la "vraie réalité ». Si la musique populaire procure une expérience qui transcende le monde, qui nous transporte «hors de nous-même»... [et] ... «nous libère des routines et attentes quotidiennes qui encombrent nos identités sociales", alors la transcendance atteinte à travers la réception de la musique «sérieuse» se produit grâce à une emphase singulière sur l'idéologie de l'autonomie, tel que l'ont constaté Leppert et McClary. Selon eux, «l'idéologie de l'auto- 
nomie informe la ... réception musicale conventionnelle du 'mélomane' qui écoute la musique dans le but précis de se détacher de la réalité et de ressentir ce qui est censé être la 'subjectivité authentique' » (Leppert et McClary 1987b: xiii).

Par ailleurs, une autre forme de l'intensification de soi dans son rapport à la société par la musique a été revendiquée par John Blacking, pour les Vendas de l'Afrique du Sud. Blacking fait l'observation suivante sur la relation entre travail et musique dans la société venda:

$\mathrm{Si}$, principalement, les Vendas jouent de la musique collectivement quand leurs estomacs sont pleins, ce n'est pas seulement pour tuer le temps ... les Vendas font de la musique quand leurs estomacs sont pleins parce que, consciemment ou inconsciemment, ils sentent les forces de séparation inhérentes à la satisfaction de la préservation de soi-même, et ils sont amenés à rétablir l'équilibre avec un comportement exceptionnellement coopératif et exploratif. Ainsi, dans la culture et la société, les forces seraient exprimées par des sons humainement organisés, parce que la fonction première de la musique dans la société et la culture est de promouvoir l'humanité sonorement organisée, en intensifiant la conscience humaine (Blacking 1973: 101).

Ce qui est sous-entendu ici, c'est que, dans la réalité quotidienne des cultures occidentales, on considère traditionnellement comme « vraiment vrai » ce qui a principalement trait aux processus fondamentaux de la reproduction matérielle de la société et de l'humanité. Selon cette perspective de la «réalité », la musique n'est pas au centre des processus sociaux ni ne possède une importance «réelle». Il s'agit, dans le cas de la musique «sérieuse» comme de la musique «populaire», d'un phénomène marginal: ou bien c'est du capital culturel, domaine du sujet indépendant et autonome, ou bien c'est du loisir et du divertissement, diversion périphérique d'occupations plus importantes qui, peut-être à cause de cette position vis-à-vis de la «réalité», permet aux individus d'éprouver une plus grande conscience d'eux-mêmes.

Dans les sociétés telles que celle des Vendas, cependant, on peut affirmer que ce qui est vu comme important et central à la culture, c'est la reproduction continuelle de la société en tant qu'unité cohésive et intégrée. Les processus de reproduction matérielle sont certes essentiels aux processus de reproduction culturelle, mais il semble reconnu que ce qui est « vraiment vrai » pour la société se situe à l'intérieur des processus de reproduction culturelle et que les processus de reproduction matérielle peuvent, éventuellement, entraîner la division. La musique, comme médium de communication unissant si puissamment et si directement l'individu et la société, est comprise, intuitivement et peut-être même consciemment, comme une force d'importance fondamentale pour la société. En manifestant et en redonnant à la société, d'une manière concrète, perceptible et reconnaissable, ses propres textures, mouvements et rythmes, la musique génère chez l'individu, 
par le biais d'une emphase sur l'intégration, un plus grand sens de lui-même. Ce plein sentiment de soi agit en retour comme le facteur premier de la reproduction culturelle. Tandis que la musique de la culture occidentale semble, dans son entier, être utilisée comme un mécanisme de retrait visant à soulager des conséquences de la réalité quotidienne, il y a plusieurs sociétés traditionnelles où la musique semble être utilisée comme un mécanisme de contemplation collective visant à réaffirmer la nature «propre » de la réalité quotidienne.

Il n'en demeure pas moins que les musiques de nos propres cultures (ainsi que celles des autres) ont des choses à nous dire que, souvent, nous n'entendons pas, à cause de la façon dont nous classons et situons socialement la musique. A ce point que les spécificités de la musique comme texte deviennent pertinentes parce que ce sont ces spécificités qu'on a gardées sous silence, soit à travers la façon dont on a traditionnellement classé et situé la musique dans nos cultures, soit encore à travers cette présomption voulant que si, en effet, la «musique » est «sociale», alors elle doit de quelque façon refléter la société plutôt que de contribuer à sa reproduction. L'aptitude de la musique à la transcendance, selon des modalités socialement constructives, son habileté à nous parler de nous-mêmes par des moyens fortement liés à ses spécificités en tant que texte, ont retenu l'attention de Catherine Ellis. La prémisse fondamentale de son exposé est que, "par la musique, nous pouvons nous déplacer vers une position plus avantageuse dans laquelle les contraires culturels [constitués, par exemple, par les Aborigènes et la société blanche] sont susceptibles de se fondre en une totalité plus grande» (Ellis 1985: 3-4). Cela est possible, selon Ellis, parce que la musique:

... peut relier une variété de processus de la pensée; elle participe à l'éducation de toute la personne; elle peut stimuler la compréhension inter-culturelle à un niveau personnel profond, de telle sorte qu'une personne n'est plus uniquement membre d'une seule culture. L'étudiant doit briser les limites de sa propre culture - souvent avec peine - et apprendre à voir toutes les situations, de plusieurs points de vue. Ce type d'apprentissage trans-culturel est à la portée de quiconque choisit d'étudier sérieusement avec des musiciens de haut calibre dont la culture est différente de la sienne. En ayant l'esprit ouvert, un étudiant peut se familiariser avec les processus de pensée de son professeur, indépendamment des barrières culturelles qui peuvent exister dans toutes les sphères d'interaction entre l'étudiant et son maître (ibidem: 15).

Pour résumer le raisonnement de Ellis, un tel apprentissage trans-culturel est réalisable parce que la musique est capable de transcender les cultures individuelles, non pas du fait qu'elle constituerait une sorte de non-culture ou un langage universel, comme on le prétend souvent en Occident, mais parce qu'elle façonne et reflète des réalités spécifiquement culturelles, et d'une manière qui échappe à la prison des modes de signification dénotatifs 
et référentiels. Si, pour les anglophones comme moi, il est difficile de connaitre la logique de la culture française, parce que nous ne pouvons pas décoder le langage, il nous est cependant possible de ressentir cette logique, d'une manière immédiate, directe et sans entraves, à travers les dimensions abstraites et relationnelles de la musique.

Le point ici soutenu [pour plus de détails, voir Shepherd 1988] est le suivant: à travers leurs dimensions abstraites et dynamiques de syntaxe, de processus et de texture, tous les types de musique peuvent parler au nexus socialement nourri et socialement constitué de l'expérience symbolique qui nous façonne tous au niveau existentiel (Shepherd 1982b). La musique, en tant que phénomène sonore, ne peut que renforcer les aspects relationnels et intégratifs de la vie humaine, soit la façon dont nous sommes en contact constant et dynamique avec le monde (Shepherd 1987). La musique permet ainsi aux Occidentaux de ressentir le monde aussi bien que de le connaître (Giles et Shepherd 1989).

La musique joue alors un rôle privilégié, et dans un sens très précis, en ce qu'elle facilite la conscience transcendante et trans-culturelle. Mais pourquoi aurions-nous besoin d'une telle prise de conscience? La réponse est liée aux fondements de notre propre socialisation. La séduction exercée par le discret, le particulier et le référentiel dans la pensée de nos cultures exclut - même si, en fin de compte, elle ne réussit pas à l'empêcher - tout mouvement par lequel nous pourrions devenir conscients des fondements de notre propre socialisation et développer l'habileté à les interroger et à les élargir de façon créative. Dans les cultures occidentales, les modes traditionnels de pensée centralisent la conscience au niveau de surface des particularités concrètes et discrètes, l'empêchant ainsi d'accéder aux niveaux structurels et relationnels plus profonds et plus abstraits constitués par les processus de socialisation. Ces modes de pensée deviennent dominants et cherchent à être exclusifs en tant que sous-produits du maintien et de la reproduction de l'hégémonie mâle et bourgeoise, à travers des modes de contrôle matériellement et visuellement médiatisés (Shepherd 1977 et 1987). Ceux qui s'enferment dans de tels modes et les font fonctionner ne désirent pas vraiment en examiner le fondement social, parce que la conscience accrue des aspects relationnels et intégratifs dans le monde humain ferait éclater comme mythe la prétendue exclusivité des systèmes de pensée basés sur le discret, le particulier et le référentiel.

Selon Ellis, la pensée, la vie et la musique des Aborigènes semblent faciliter l'accès aux fondements de la socialisation, d'une part, parce que les limites, non conçues de manière rigide, sont constamment adaptables au changement négocié et, d'autre part, parce que ces mêmes limites, une fois acceptées et définies, peuvent s'accommoder à différentes acceptions de contenu. Un tel système culturel - qui semble permettre l'accès aux fondements de la 
socialisation à ceux qui sont vieux (40 ans et plus), sages, et sur lesquels on peut compter pour utiliser pareil pouvoir de manière constructive et non destructive - pourrait alors s'ajuster à certaines prémisses d'autres systèmes culturels. Cet accès peut passer - et passe souvent - par la musique. Grâce à ses dimensions abstraites et dynamiques de syntaxe, de processus et de texture, la musique semble, de tous les médias de communication, la plus apte à parler directement et globalement à ces processus abstraits qui donnent aux gens les fondements de leur socialisation. On pourrait dire que la musique convient idéalement au rassemblement des fondements de la socialisation et à la production d'une expérience très puissante dans la mesure où elle n'a pas à s'appuyer, en dernière instance, sur le spécifique et le particulier. Cependant, la société aborigène court deux dangers en s'ouvrant aux fondements de son propre système culturel et à ceux des autres. Premièrement, il y a toujours le danger que quelqu'un utilise le pouvoir secret du chant de façon erronée:

Les lourdes sanctions qui s'appliquent à l'enseignement de chants secrets et à l'utilisation du pouvoir qu'ils contiennent, sont la déclaration d'une limite fixe qui, si elle est traversée par la mauvaise personne, peut causer la désintégration du système par l'intérieur. Une fois que la personne initiée a été admise dans de tels domaines de pouvoir, elle ne peut subséquemment être exclue (Ellis 1985: 87).

Deuxièmement, il y a le danger de l'incursion d'une nature importune provenant de l'extérieur:

Dans un tel système, où l'inclusion est toujours possible, il est nécessaire d'avoir des barrières solides pour prévenir l'intrusion malsaine. Un trop haut degré d'ingérence non traditionnelle peut forcer le système à un point au-delà duquel il ne puisse conserver ses limites intactes. Cette éventualité méconnue d'un excès d'interférence dans le contact tribal/blanc mène souvent à une effondrement total du système tribal (ibidem: 85 ).

En ce qui nous concerne, nous ne nous mettons dans aucune de ces situations dangereuses. Soit que nous ayons perdu l'habileté à recueillir, à travers la musique, un pouvoir social pleinement intégré, soit que nous ne reconnaissions pas ce pouvoir ou que ne voulions pas le reconnaître pour ce qu'il est au moment où nous le ressentons. D'une façon ou d'une autre, nous ne semblons pas capables de bénéficier d'un tel pouvoir d'une manière pleinement transcendantale.

\section{Conclusion}

Nous pouvons maintenant revenir à la question soulevée, il y a plus de vingt ans, dans l'échange entre Harrison et Kerman. La distinction faite par 
Kerman, lorsqu'il dit qu'il serait plus profitable d'étudier les personnes en société dans le but d'élargir la compréhension des œuvres d'art que d'étudier les œuvres d'art afin de mieux comprendre les personnes en société, est d'une importance cruciale pour la musicologie: en effet, la musicologie ne devrait jamais devenir une projection de la sociologie, fournissant à la sociologie, et en des termes dictés par les différentes problématiques de cette discipline, les informations et les preuves nécessaires à la compréhension des processus sociaux.

Il se peut que, dans le contexte du type de critique fait par Swanwick en ce qui concerne mes propres recherches, l'insistance de Frith - selon laquelle les questions d'esthétique devraient être approchées sur une base sociologique - s'avère justifiée. Toute discussion sur la musique devrait être nourrie par la sociologie. Même si, comme Kerman, l'on défend l'idée que, dans le domaine de la critique musicologique, toutes les analyses sociologiques et musicologiques de caractère technique devraient se soumettre à l'entreprise critique, il ne s'ensuit pas nécessairement qu'une critique musicologique sociologiquement fondée serait, de son côté, incapable d'apporter des points de vue pénétrants sur les conditions de notre culture et de celle des autres. C'est peut-être en ce sens que se justifie la revendication de Harrison selon laquelle la musicologie devrait devenir l'étude des personnes en société, «dans la mesure où elles s'expriment par l'intermédiaire de la musique ». Au lieu de tenter de devenir une projection de la sociologie (ou de l'anthropologie) ou, pis encore, de demeurer tout simplement une entreprise isolée, la musicologie critique devrait s'ouvrir à l'éventualité de remplir un rôle distinct et spécifique, lequel consisterait à élargir notre compréhension des différentes cultures et sociétés.

Récemment, Kerman se plaignait:

Ce que j'appellerais la critique musicale sérieuse - la critique musicale académique, si vous préférez — n'existe pas en tant que discipline au même niveau que la musicologie et la théorie musicale d'un côté, ou la critique littéraire et artistique de l'autre. Nous n'avons pas nos Arnold ou nos Elliot de la musique, pas davantage de Blackmur ou Kermode, non plus que de Ruskin ou de Schapiro. Dans ces circonstances, il est vain de se plaindre que la pensée critique, en musique, se trouve conceptuellement loin derrière les autres arts. En fait, la plupart des penseurs de la musique voyagent à une distance respectueuse derrière les derniers chars de la vie intellectuelle en général . . . La sémiologie, l'herméneutique et la phénoménologie ne sont aujourd'hui connues que grâce à certaines des études musicales les plus osées. Le post-structuralisme, la déconstruction et le féminisme sérieux ont encore à faire leurs débuts en musicologie et en théorie musicale (Kerman 1985: 17).

En s'ouvrant à l'éventualité d'apporter une contribution distincte et 
spécifique à la compréhension des différentes cultures et sociétés, la musicologie pourrait sûrement prendre sa place dans le monde académique en tant que discipline, à côté d'autres disciplines dont la conscience critique est beaucoup plus développée. Le problème n'est plus de savoir comment comprendre la musique et les individus en termes sociaux. La question cruciale est devenue: comment comprendre les sociétés et les individus en termes musicaux? Ce qu'il faut développer n'est plus une sociologie et une esthétique de la musique, mais une musicologie de l'esthétique et de la société.

Ellis présente donc un message opportun à propos de l'importance et l'usage approprié de la musique. Ce message propose une structure destinée à faire évoluer la critique et l'évaluation de la musique au-delà de ce qui a été envisagé par Kerman ou par Frith. Cependant, pour que cette structure ait la chance de se développer, il est nécessaire de bien situer la ligne de pensée de Ellis, et ses critiques éventuelles, dans les circonstances sociales et historiques du capitalisme avancé. La récupération appropriée de la musique est particulièrement importante pour notre société, à cause de ce qu'elle peut nous apprendre de nous-mêmes et de notre avenir. Si la musicologie, en tant que discipline, ne reconnaît pas cela, elle continuera alors, comme son objet d'étude, à occuper une position marginale et périphérique dans le monde, répondant toujours aux initiatives intellectuelles des autres. Pour reprendre les mots de Arthur Mendel, elle restera «une science jeune» (1962: 14). Les musicologues n'ont pas seulement besoin d'analyser, de déconstruire, de creuser sous la surface de la réalité, d'aider à comprendre la reproduction musicale et culturelle de notre propre monde. Les musicologues ont besoin de regarder au-delà aussi bien qu'au-dessous de la réalité; à travers une compréhension plus poussée de ce que la musique peut nous révéler de nous-mêmes et de notre propre société, ils doivent établir des stratégies de changement dans les modes de conscience et les relations interhumaines. Ellis donne une impulsion à ces initiatives, nous rappelant ainsi que, comme toute autre activité humaine, la musicologie est une entreprise essentiellement politique.

(Traduction de Sylvie Roulx)

\section{RÉFÉRENCES}

BLACKING, John

1973: How Musical is Man? Seattle: University of Washington Press.

ELLIS, Catherine

1985: Aboriginal Music: Education for Living. St. Lucia: University of Queensland Press. 
FRITH, Simon

1987: "Towards an Aesthetic of Popular Music," dans Leppert et McClary 1987a, 133-149.

GILES, Jennifer

1987: Music Consumption among English Speaking Teenage Girls in the City of Montreal. Mémoire de maîtrise, Institute of Canadian Studies, Carleton University.

GILES, Jennifer et SHEPHERD, John

1989: “Theorizing Music's Affective Power" Arena, 85, 106-121.

HARRISON, Frank L1.

1963: "American Musicology and the European Tradition," dans Musicology écrit en collaboration avec Mantle Hood et Claude V. Palisca. Englewood Cliffs, New Jersey: Prentice-Hall.

KERMAN, Joseph

1965: "A Profile for American Musicology," Journal of the American Musicological Society, 18, 61-69.

1985: Contemplating Music: Challenges to Musicology. Cambridge, Mass.: Harvard University Press.

LEPPERT, Richard et McCLARY, Susan (édit.)

1987a: Music and Society: The Politics of Composition, Performance and Reception. Cambridge: Cambridge University Press.

1987b: “Introduction," dans Leppert et McClary 1987a, xi-xix.

MENDEL, Arthur

1962: "Evidence and Explanation," Report of the Eighth Congress of the International Musicological Society, New York. Cassel, London et New York : Barenreiter.

MEYER, Leonard B.

1959: "Some Remarks on Value and Greatness in Music," Journal of Aesthetics and Art Criticism, 17, 486-500.

1973: Explaining Music. Los Angeles: University of California Press.

PALISCA, Claude

1963: "American Scholarship in Western Music," dans Musicology, écrit en collaboration avec Frank Ll. Harrison et Mantle Hood. Englewood Cliffs, New Jersey: Prentice-Hall.

SHEPHERD, John

1977: "Media, Music and Social Process," "The 'Meaning' of Music" et "The Musical Coding of Ideologies," dans Whose Music? A Sociology of Musical Languages, par Shepherd et al. London: Latimer; réédité chez Transaction (1980). 
1982a: "A Theoretical Model for the Sociomusicological Analysis of Popular Musics," Popular Music, 2, 145-177.

1982b: "R. D. Laing and the Social Construction of Self: A Theoretical Speculation," Human Affairs, vol. 2 no 1, 1-17.

1985: "Prolegomena for the Critical Study of Popular Music," Canadian Journal of Communications, vol. 11, no 1, 17-34; 141-144.

1987: "Music and Male Hegemony," dans Leppert et McClary 1987a, $151-172$.

1988: “Towards a Musicology of Society," Echology, no 2, 101-124.

SUBOTNIK, Rose Rosengard

1982: "Musicology and Criticism," dans Musicology in the 1980's, Kern Holoman et Claude Paslica (édit.). New York: Da Capo, 145-160.

SWANWICK, Keith

1984: "Problems of a Sociological Approach to Pop Music in Schools," British Journal of Sociology of Education, vol. 5, no 1, 49-56.

VULLIAMY, Graham et SHEPHERD, John

1984: "Sociology and Music Education: A Response to Swanwick," British Journal of Sociology of Education, vol. 5, no 1, 57-76. 